\title{
Evaluation of Blood Loss and Implant Alignment after Total Knee Arthroplasty with Inertial Based Extramedullary Femoral Cutting Guide
}

\author{
Tommaso Bonanzinga ${ }^{1,2}$ Piergiuseppe Tanzi ${ }^{3}$ Maria Pia Neri ${ }^{3}$ Francesco lacono ${ }^{1,2}$ Claudio Mazzola ${ }^{4}$ \\ Alberto Belluati $^{5}$ Andrea Colombelli ${ }^{5}$ Stefano Zaffagnini ${ }^{3}$ Maurilio Marcacci ${ }^{1,2}$
}

${ }^{1}$ Department of Biomedical Sciences, Humanitas University, Milan, Italy

2 Humanitas Clinical and Research Center, Milan, Italy

${ }^{3}$ Rizzoli Orthopedic Institute, Bologne, Italy

${ }^{4}$ Hospital Galliera, Genoa, Italy

${ }^{5}$ Complex Unit of Orthopedic Hospital of Ravenna, Ravenna, Italy

Address for correspondence Tommaso Bonanzinga, MD, Humanitas Clinical and Research Center, Building 8, Via Manzoni 113, 20089 Rozzano, Milan, Italy (e-mail: t.bonanzinga@gmail.com).

Joints 2018;6:161-166.

\begin{abstract}
Keywords

- total knee arthroplasty

- extramedullary guide

- blood loss

- alignment

- cutting guides

Purpose The purpose of this study was to compare, in terms of blood loss and implant alignment, a new generation of smart extramedullary (EM) cutting guides with the conventional intramedullary (IM) guide for total knee arthroplasty (TKA). The hypothesis was that the EM system would result in less blood loss and fewer days of hospitalization, while ensuring equal or higher accuracy in the alignment of the femoral implant.

Methods Thirty-six patients were enrolled for the present study: 18 patients underwent TKA using the EM guide and for the other 18 patients the IM guide was used. Preoperative and postoperative $\mathrm{X}$-rays were acquired. The preoperative and postoperative hemoglobin values were compared. Lastly, length of hospital stay was recorded.

Results The hemoglobin difference was significantly lower in the EM group. The alignment of the prosthetic femoral implant in the two groups was comparable but all patients in the EM cohort had a final alignment within 3 degrees of range on the frontal view, while $22 \%$ of the patients in the IM cohort had a final alignment exceeding 3 degrees of range. The mean hospitalization duration showed no significant difference between groups, though EM group showed 1.6 days less compared with IM group.

Conclusions The inertial-based EM guide system could be useful for decreasing blood loss compared with conventional guide. This device tended to have better results even in terms of accuracy, but this difference was not significant.

Level of Evidence This is a level II, prospective cohort study.
\end{abstract}

\section{Introduction}

Proper alignment of femoral, tibial, and patellar components is critical for implant survival of total knee arthroplasty (TKA). The desired alignment of the femoral component is traditionally obtained with the use of an intramedullary (IM) guide, but recent reports showed that IM guides achieve correct femoral component alignment, within less than 2-degree error, in less than $66 \%$ of knees. ${ }^{1,2}$ Moreover, the use of IM guides implies risks for the patients, such as perioperative blood loss and the requirement for blood transfusion after joint replacement. Kim et $\mathrm{al}^{3}$ showed that the invasiveness of IM guide leads to thromboembolic complications in $4 \%$ of cases with subsequent perioperative morbidity and neurological dysfunctions. Kalairajah et $\mathrm{al}^{4}$ reported a significant reduction in blood drainage and received

November 18, 2017 accepted after revision August 26, 2018 published online October 18, 2018
DOI https://doi.org/

10.1055/s-0038-1673404. ISSN 2282-4324.
Copyright $\odot 2018$ Georg Thieme Verlag License terms KG Stuttgart · New York 
hemoglobin loss with the use of computer-assisted surgery (CAS) in comparison with the conventional techniques. This allows reduced risks of transfusion and financial saving. Kandel et $\mathrm{al}^{5}$ reported that extramedullary (EM) femoral alignment instrumentation reduces blood loss after cementless TKA compared with IM device. In recent years, EM accelerometer-based devices were developed to improve accuracy of implant positioning ${ }^{6-9}$ and to reduce patient morbidity. These devices have inertial electronic components attached to the femoral and tibial resection instruments, which enable surgical resection correctly aligned in the coronal and sagittal planes without damage to the IM canal and associated risks, such as blood loss. Fang et $\mathrm{al}^{10}$ showed that a well-positioned TKA leads to good clinical results and that malalignment is a predictor for TKA revision surgery (varus knees failed primarily by medial tibia collapse, whereas valgus knees failed from ligament instability).

The purpose of the present study was to compare, in terms of blood loss and implant alignment in the coronal and sagittal planes, a new generation of smart EM cutting guides with the conventional technique based on the use of IM guides. The hypothesis was that the EM system, by avoiding the medullary breaching, would result in less blood loss and less hospitalization stay due to the better postoperative clinical status and hemoglobin values, while ensuring equal or even higher accuracy in the alignment of the femoral implant.

\section{Methods}

\section{Study Design}

The authors conducted a comparative multicenter randomized study on patients undergoing TKA at two independent centers in which one surgeon for center performed all the surgical procedures. The ethics committees of each center approved the study in advance.

\section{Patients}

The inclusion criteria were: patients undergoing primary TKA and age between 60 and 75 years; exclusion criteria were: pathological conditions affecting the ipsilateral knee or ankle, previous surgery on the ipsilateral hip or ankle, intra- or extra-articular deformities limiting the use of the regular IM guide, systemic disease affecting the hemoglobin levels in blood, or the patients' erythropoietic potential, and anticoaugulation or antiplatelet therapy.

Thirty-six patients were enrolled (18 patients in each unit). After obtaining a written informed consent, patients were randomly assigned a randomization online software (www. randomization.com) to either of the two study groups 18 patients underwent TKA using the novel smart EM cutting guide (Perseus, Orthokey Italia, Italy; group A) while in the other 18 patients the conventional IM guide was used (group B).

\section{Interventions}

The same surgical approach (parapatellar medial capsulotomy) under tourniquet was used in the two groups. Both centers used the same implant (Attune; Depuy, Warsaw, IN, U.S.A.) and all the implants were cemented. Balance of space in flexion and extension (gap balancing technique) was used in both groups.

In the experimental group $(\mathrm{A})$, the femoral distal resection was performed using the EM system based on inertial sensors, while in the control group (B) the femoral distal resection was performed using conventional IM instruments.

The Perseus EM guide consists of a cutting guide assembled with a disposable device containing inertial sensors that communicate via Bluetooth with a tablet placed outside the surgical field ( - Fig. 1). After performing the surgical approach, Perseus is fixed to the bone segment close to the resection area (-Fig. 2). To determine the anatomical reference axes, the angular relationship between the electronic pod of the digitizer

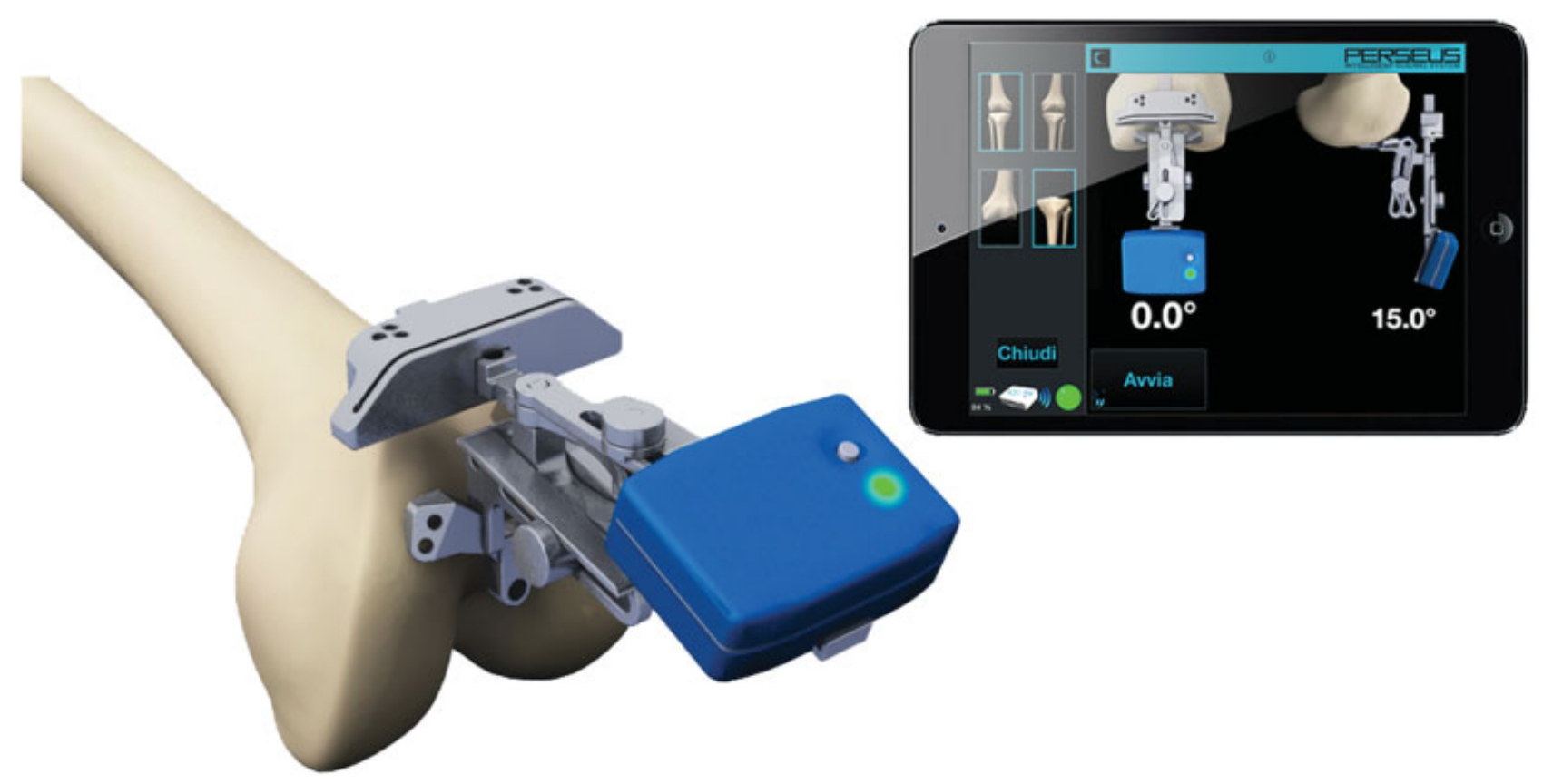

Fig. 1 Perseus cutting guide. 


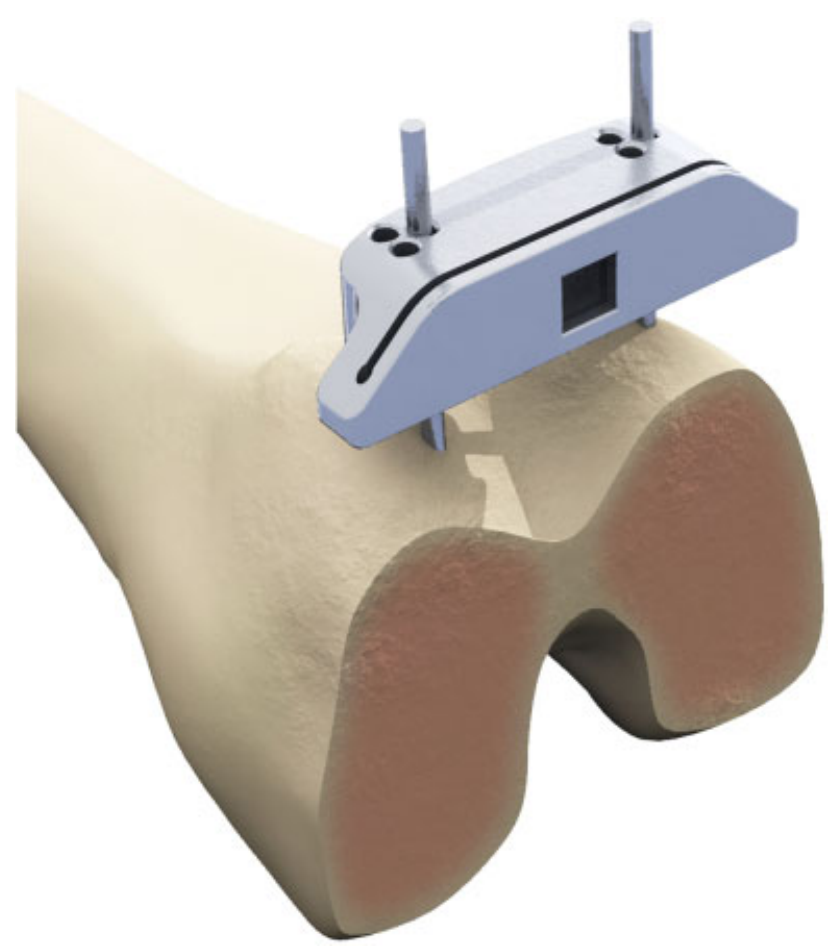

Fig. 2 Perseus fixed to bone segment close to resection area.

and the bone reference is registered by the system through movements of the hip: abduction-adduction and flexionextension. This function activates the inertial mechanism, which then identifies the femoral mechanical axis. Starting from this axis, the triaxial inertial device directs the surgeon on how to align the cutting guide for a correct performance of the resection; plane control was on frontal and sagittal planes (-Fig. 3).

The two groups of patients underwent the same postoperative protocol for rehabilitation, antithromboembolic prophylaxis (enoxaparin 4,000 IU/die) and pain medications.

\section{Outcome Measurements}

Preoperative and postoperative (at discharge) nonweightbearing X-rays were acquired in anteroposterior (AP), lateral,

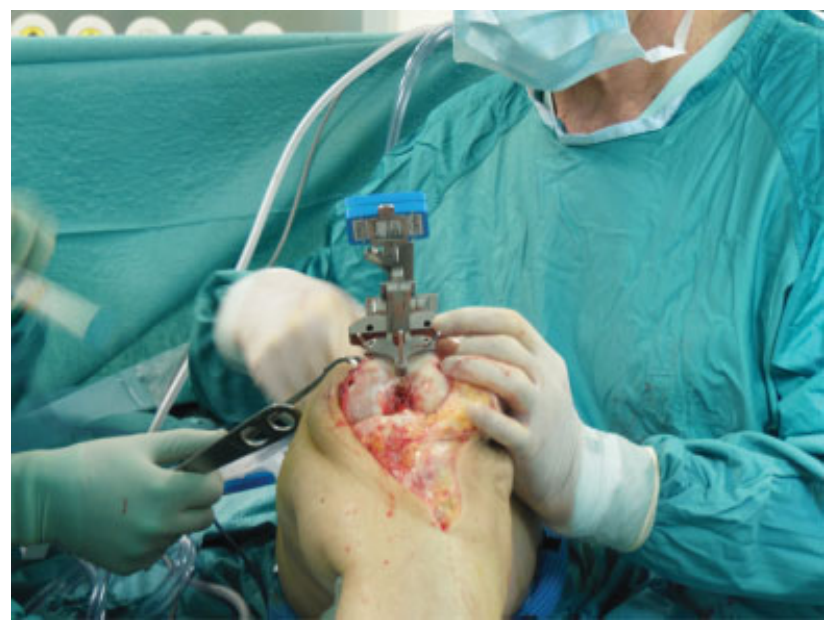

Fig. 3 Surgical image of femoral cut using extramedullary guide.
Table 1 Baseline characteristics of patients of the two groups

\begin{tabular}{|l|l|l|l|l|}
\hline Group & Age (y) & $\begin{array}{l}\text { Sex } \\
(\mathbf{m} / \mathbf{f})\end{array}$ & $\begin{array}{l}\text { Limb preop } \\
\text { alignment (degree) }\end{array}$ & $\begin{array}{l}\text { Hemoglobin } \\
(\mathbf{g} / \mathbf{d L})\end{array}$ \\
\hline A (EM) & $71 \pm 5$ & $10 / 8$ & $4.0 \pm 2.5$ (varus) & $12.0 \pm 1.7$ \\
\hline B (IM) & $72 \pm 6$ & $6 / 12$ & $4.6 \pm 4.1$ (varus) & $12.7 \pm 1.4$ \\
\hline
\end{tabular}

Abbreviations: EM, extramedullary; IM, intramedullary; preop, preoperative.

and full-limb views, taking care to position the limb in a neutral position. Two independent investigators measured the angles between the prosthetic femoral component and the femoral mechanical axis on the frontal plane and the anterior cortex on the lateral plane. Hemoglobin values were recorded during hospitalization and preoperative values were compared with the minimum postoperative value obtained. Lastly, length of stay (LOS) in hospital was recorded.

\section{Statistical Analysis}

Due to the sample size and nonuniform data distribution, the Kruskal-Wallis test was used to compare differences between the two groups in terms of blood loss and implant alignment. Sample size calculation was based on the existing literature, which shows that the average and the standard deviation of the variation of hemoglobin following TKA performed with the conventional IM technique are equal to $3.5 \mathrm{~g} / \mathrm{dL}$ and $1.2 \mathrm{~g} / \mathrm{dL}$, respectively. Assuming that the alternative treatment produces at least a reduction of $1 \mathrm{~g} / \mathrm{dL}$ hemoglobin level and assuming an $\alpha$ level equal to 0.05 and a power of at least $80 \%$, the minimum number of cases per group required was 18 (total number: 36 ).

\section{Results}

- Table 1 shows complete baseline data. No differences were observed in terms of age, preoperative limb alignment, or preoperative hemoglobin level in two groups.

Estimate of blood loss showed differences between the two groups (-Table 2 ). The hemoglobin difference was significantly lower in the EM group $(p=0.0371)$.

The results of the alignment of the prosthetic femoral implant are reported in -Table 3. Perpendicularity of the distal resection to the femoral mechanical axis was comparable in the two groups. All patients in the EM cohort had a final alignment within 3 degrees of range on the frontal view,

Table 2 Hemoglobin variation in the two groups

\begin{tabular}{|l|l|l|l|}
\hline \multirow{2}{*}{ Group } & \multicolumn{3}{|l|}{ Hemoglobin (g|dL) } \\
\cline { 2 - 4 } & Preop (min) & Postop (min) & Variation \\
\hline A (EM) & $12.0 \pm 1.4$ & $9.3 \pm 0.9$ & $2.4 \pm 1.3^{\mathrm{a}}$ \\
\hline B (IM) & $12.7 \pm 1.7$ & $9.5 \pm 1.3$ & $3.3 \pm 1.2$ \\
\hline
\end{tabular}

Abbreviations: EM, extramedullary; IM, intramedullary; preop, preoperative; postop, postoperative.

${ }^{\mathrm{a} S}$ Significant difference. 
Table 3 Results related to angular deviation of the prosthetic femoral implant

\begin{tabular}{|l|l|l|}
\hline \multirow{2}{*}{ Group } & \multicolumn{2}{|l|}{ Angular deviation (degree) } \\
\cline { 2 - 3 } & Frontal plane & Sagittal plane \\
\hline A (EM) & $0.6 \pm 1.1$ (varus) & $-0.6 \pm 1.9$ (extension) \\
\hline B (IM) & $0.7 \pm 2.8$ (varus) & $2.2 \pm 4.4$ (extension) \\
\hline
\end{tabular}

Abbreviations: EM, extramedullary; IM, intramedullary.

while four patients (22\%) in the IM cohort had a final alignment exceeding 3 degrees of range (-Fig. 4). Lastly, the mean LOS in hospital showed no significant difference between groups, though group (A) showed 1.6 days less compared with group (B) $(7.7 \pm 3.1$ and $9.3 \pm 3.9$ days for group $\mathrm{A}$ and $\mathrm{B}$, respectively).

\section{Discussion}

The main finding of this study was that blood loss was lower in patients who underwent TKA with the EM guide, compared with the conventional IM technique.

Blood loss during TKA is an important issue that is often underestimated. Patients who undergo this type of surgery are usually over the age of 60 years and concomitant patho-

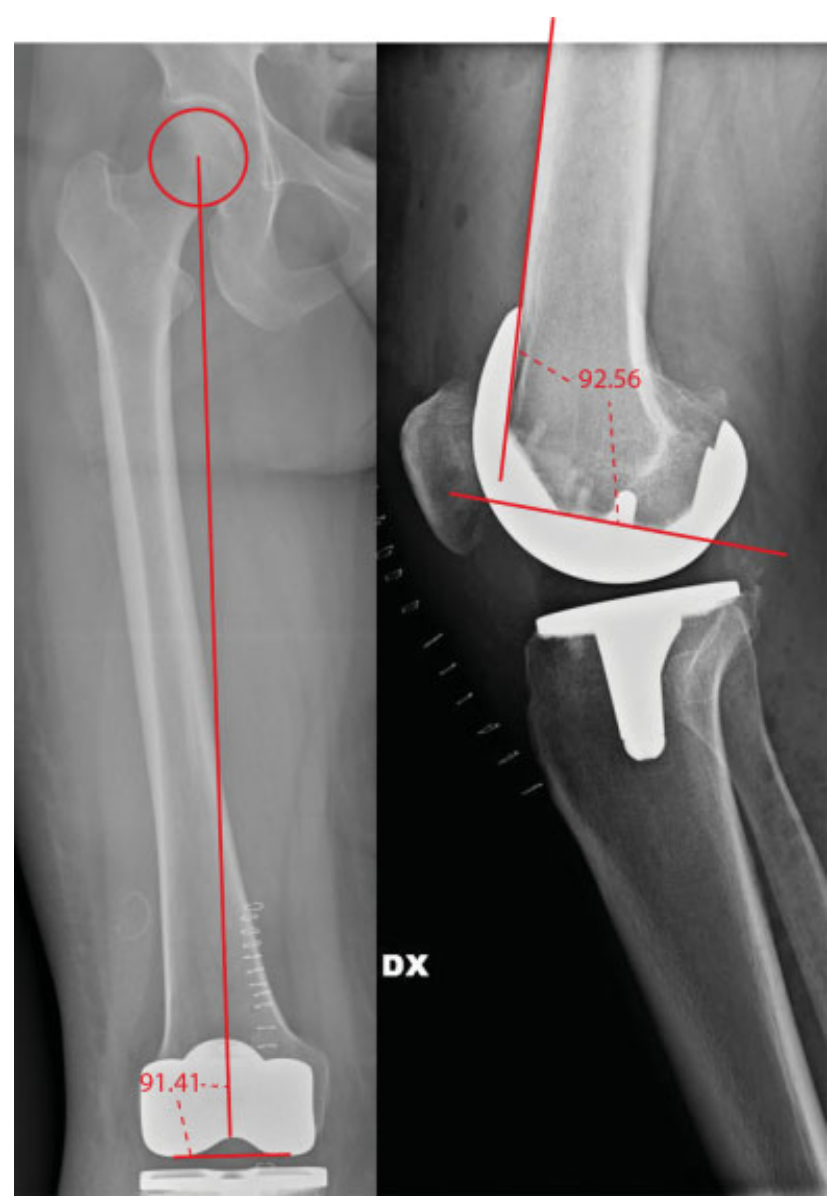

Fig. 4 Postoperative X-ray: proper alignment on the frontal and sagittal plane. logical conditions such as hypertension, diabetic disorders, and heart diseases are often observed. ${ }^{11}$ Less blood loss may help to reduce the intraoperative and postoperative risks. Furthermore, significant blood loss may require a blood transfusion, which carries its own risks including infection, abnormal immunological responses, febrile transfusion reactions, and allergic reactions. ${ }^{12}$

This study found that variation in the hemoglobin value during hospitalization was of $2.4 \pm 1.3$ in group (A) and $3.3 \pm 1.2$ in group (B). This latter difference was statistically significant. These results are comparable with those of other studies $^{4,5}$ that showed blood loss could be reduced when the medullary canal was not breached during TKA. Jeon et al ${ }^{12}$ compared the EM and conventional IM femoral alignment guide systems for bleeding and transfusion rate after TKA and demonstrated that the EM femoral alignment guide technique was advantageous in reducing the volume of blood drained and the allogeneic transfusion rate. In a similar setup, Kandel et $\mathrm{al}^{5}$ reported a significant lower average blood drainage volume and transfusion rate using EM guide as well. Using a navigation system to align the femoral component, Kalairajah et $\mathrm{al}^{4}$ reported significantly lower hemoglobin loss compared with the conventional procedure.

Implant positioning in the EM group was comparable to IM instrumentation in the final alignment but showed a reduced distribution within the average value, especially in the sagittal plane. On the frontal plane, no patients operated on with EM guide were outside 3 degrees of alignment range, while $22 \%$ in the IM cohort had a final alignment outside 3 degrees of range. Although recent studies ${ }^{13,14}$ questioned the role of overall TKA postoperative alignment in implant survival, most of the authors ${ }^{1,2,10,15}$ still advocate placing the implant within 3 degrees of a neutral mechanical axis. Fang et $\mathrm{al}^{10}{ }^{10}$ in their retrospective study, based on 6,070 TKA patients looking for implant survival in well-aligned knees compared with that of the outliers (more than 3 degrees of valgus or varus alignment) reported that well-aligned TKAs has a longer survival than ones placed in varus or valgus alignment. Wear of the polyethylene tibial bearing surface is a leading cause of failure in knee replacements performed in the last decade due to limb malalignment, patient-related, and implant-related factors. ${ }^{16}$

In the present study, EM guide demonstrated proper distal cut alignment with respect to the femoral mechanical axis on the frontal plane. Patients operated with EM guide were all inside the alignment range (100\%), whereas the standard alignment in the IM group was observed in 78\% of the cases. Nam et $\mathrm{al}^{7}$ using a different inertial-based EM guide system reported no significant difference in the number of TKAs within 3 degrees of a neutral mechanical alignment compared with a conventional IM system in a cohort of 94 patients. However, a trend was seen in the EM cohort toward a higher percentage of implants being in the correct alignment range $(89.4 \%$ vs. $74.5 \%)$. Scuderi et $\mathrm{al}^{6}$ described the results of another EM inertial-based guide. The authors included 25 patients and reported results from the first 14 . The femoral mechanical axis showed an average accuracy of $0.4 \pm 0.95$ between the confirmed bone surface and the postoperative computed tomography (CT). 
A clear advantage of the smart EM cutting guides is the easy to use and the shorter surgical time compared with CAS. Navigation systems is more complex to setup compared with the EM guide as only one pin is required to close the system and the acquisition process is quick and easy. Goh et $\mathrm{al}^{17}$ included 76 patients and compared the results of the EM inertial-based guide and CAS. They reported no significant difference in clinical outcomes between the two groups. The duration of surgery, however, was significantly longer in the CAS group (101 \pm 11 minutes), compared with the EM group ( $84 \pm 21$ minutes). The percentage of patients with an alignment within 3 degrees of a neutral mechanical axis for the EM group was $92.1 \%$, which was comparable to the CAS group and to other studies of largeconsole CAS systems. ${ }^{11,18-21}$ The authors found reproducible accuracy in component alignment with inertial-based femoral resection.

Another crucial aspect in the positioning of the femoral component is flexion in the sagittal plane. In the present study, the EM cutting guide proved better in terms of flexion of the femoral component, but again this difference was not significant. Kim et $\mathrm{al}^{15}$ found a connection between sagittal femoral component alignment and early component failure. Out of 3,048 knees investigated at 15.8 years, 3.3\% of flexed required revision while no neutrally aligned femoral implants required revision. Maderbacher et $\mathrm{al}^{2}$ found that IM alignment rods caused a mean sagittal alignment of the cutting block of 4.4 degrees of flexion in relation to the mechanical femoral axis. In only $25 \%$ of the observed cases, a flexion within a range of the desired value of 0 to 3 degrees was obtained. The authors concluded that exact sagittal femoral component alignment could only be achieved by navigation devices. In the current study there was no significant difference between EM and the IM cutting guide; however, once again EM guide tended to perform better.

The present study found that, using EM guide, fewer days of hospitalization were necessary compared with patients who underwent TKA using conventional IM guide, albeit this difference was not statistically significant.

The present study has some limitations. First, the choice of using traditional X-rays in place of CT implies possible measurement errors due to limb rotation. Standing AP hip-to-ankle radiographs and lateral knee-to-ankle radiographs were used. It is possible that $\mathrm{CT}$ is a more accurate radiologic method, as radiographs can be subject to rotational variations. However, CT has several disadvantages, including radiation exposure and metallic artifacts, and hip-to-ankle radiographs are easily obtained in the routine follow-up, avoiding an increase in the costs of the study. Radiographers were trained to obtain consistent films, and radiographs were repeated if malrotation was detected. Further, it has been shown that radiographs are reproducible for assessing implant placement ${ }^{22,23}$ and have less exposition to radiation. The second limitation was the low number of patients and the lack of follow-up. Lastly, it is difficult to draw conclusions from this study regarding the "learning curve" that is required in the use of the accelerometer-based navigation device. The inclusion period took a considerable amount of time, and surgeons in the study would often go for several months without using this technique. However, a previous cadaveric study has demonstrated a rapid learning curve with inertial-based cutting guides. ${ }^{19}$

In conclusion, the inertial-based EM guide system could be useful to decrease blood loss in TKA and to obtain better postoperative clinical conditions and shorter hospital stay compared with conventional IM guide systems. This device tended to have better results even in terms of accuracy of implant alignment, but these differences were not significant. Future studies are needed to compare the performance of Perseus with other inertial-based systems, which are generally easier to use than navigation systems and less invasive than conventional IM guide systems.

\section{Conflict of Interest}

None declared.

\section{References}

1 Ritter MA, Davis KE, Meding JB, Pierson JL, Berend ME, Malinzak RA. The effect of alignment and BMI on failure of total knee replacement. J Bone Joint Surg Am 2011;93(17):1588-1596

2 Maderbacher G, Schaumburger J, Baier C, et al. Appropriate sagittal femoral component alignment cannot be ensured by intramedullary alignment rods. Knee Surg Sports Traumatol Arthrosc 2016;24 (08):2453-2460

$3 \mathrm{Kim} \mathrm{YH}$. Incidence of fat embolism syndrome after cemented or cementless bilateral simultaneous and unilateral total knee arthroplasty. J Arthroplasty 2001;16(06):730-739

4 Kalairajah Y, Simpson D, Cossey AJ, Verrall GM, Spriggins AJ. Blood loss after total knee replacement: effects of computer-assisted surgery. J Bone Joint Surg Br 2005;87(11):1480-1482

5 Kandel L, Vasili C, Kirsh G. Extramedullary femoral alignment instrumentation reduces blood loss after uncemented total knee arthroplasty. J Knee Surg 2006;19(04):256-258

6 Scuderi GR, Fallaha M, Masse V, Lavigne P, Amiot LP, Berthiaume MJ. Total knee arthroplasty with a novel navigation system within the surgical field. Orthop Clin North Am 2014;45(02):167-173

7 Nam D, Cody EA, Nguyen JT, Figgie MP, Mayman DJ. Extramedullary guides versus portable, accelerometer-based navigation for tibial alignment in total knee arthroplasty: a randomized, controlled trial: winner of the 2013 HAP PAUL award. J Arthroplasty 2014;29(02):288-294

8 Nam D, Jerabek SA, Haughom B, Cross MB, Reinhardt KR, Mayman DJ. Radiographic analysis of a hand-held surgical navigation system for tibial resection in total knee arthroplasty. J Arthroplasty 2011;26(08):1527-1533

9 Nam D, Nawabi DH, Cross MB, Heyse TJ, Mayman DJ. Accelerometer-based computer navigation for performing the distal femoral resection in total knee arthroplasty. J Arthroplasty 2012;27(09):1717-1722

10 Fang DM, Ritter MA, Davis KE. Coronal alignment in total knee arthroplasty: just how important is it? J Arthroplasty 2009;24(6, Suppl):39-43

11 Heidari B. Knee osteoarthritis prevalence, risk factors, pathogenesis and features: Part I. Caspian J Intern Med 2011;2(02):205-212

12 Jeon SH, Kim JH, Lee JM, Seo ES. Efficacy of extramedullary femoral component alignment guide system for blood saving after total knee arthroplasty. Knee Surg Relat Res 2012;24(02):99-103

13 Parratte S, Pagnano MW, Trousdale RT, Berry DJ. Effect of postoperative mechanical axis alignment on the fifteen-year survival of modern, cemented total knee replacements. J Bone Joint Surg Am 2010;92(12):2143-2149

14 Sikorski JM. Alignment in total knee replacement. J Bone Joint Surg Br 2008;90(09):1121-1127 
Bonanzinga et al.

15 Kim YH, Park JW, Kim JS, Park SD. The relationship between the survival of total knee arthroplasty and postoperative coronal, sagittal and rotational alignment of knee prosthesis. Int Orthop 2014;38(02):379-385

16 Collier MB, Engh CA Jr, McAuley JP, Engh GA. Factors associated with the loss of thickness of polyethylene tibial bearings after knee arthroplasty. J Bone Joint Surg Am 2007;89(06): 1306-1314

17 Goh GS, Liow MH, Lim WS, Tay DK, Yeo SJ, Tan MH. Accelerometerbased navigation is as accurate as optical computer navigation in restoring the joint line and mechanical Axis after total knee arthroplasty: a prospective matched study. J Arthroplasty 2016; 31(01):92-97

18 Mason JB, Fehring TK, Estok R, Banel D, Fahrbach K. Meta-analysis of alignment outcomes in computer-assisted total knee arthroplasty surgery. J Arthroplasty 2007;22(08):1097-1106

19 Nam D, Dy CJ, Cross MB, Kang MN, Mayman DJ. Cadaveric results of an accelerometer based, extramedullary navigation system for the tibial resection in total knee arthroplasty. Knee 2012;19(05): 617-621

20 Nam D, Weeks KD, Reinhardt KR, Nawabi DH, Cross MB, Mayman DJ. Accelerometer-based, portable navigation vs imageless, largeconsole computer-assisted navigation in total knee arthroplasty: a comparison of radiographic results. J Arthroplasty 2013;28(02): 255-261

21 Sparmann M, Wolke B, Czupalla H, Banzer D, Zink A. Positioning of total knee arthroplasty with and without navigation support. A prospective, randomised study. J Bone Joint Surg Br 2003;85 (06):830-835

22 Duparc J, Massare C. [Radiological measurement of the angular deviation of the knee in the frontal plane]. Ann Radiol (Paris) 1967;10(09):635-656

23 Spencer JM, Chauhan SK, Sloan K, Taylor A, Beaver RJ. Computer navigation versus conventional total knee replacement: no difference in functional results at two years. J Bone Joint Surg $\mathrm{Br}$ 2007;89(04):477-480 